Article

\title{
Thermal Stability of Modified Insulation Paper Cellulose Based on Molecular Dynamics Simulation
}

\author{
Chao Tang ${ }^{1, *}$, Song Zhang ${ }^{1}$, Qian Wang ${ }^{2}$, Xiaobo Wang ${ }^{1}$ and Jian Hao ${ }^{3}$ \\ 1 College of Engineering and Technology, Southwest University, Chongqing 400715, China; \\ xbf_1992@sina.cn (S.Z.); wangxiaobo666999@163.com (X.W.) \\ 2 State Grid Chongqing Electric Power Co. Chongqing Electric Power Research Institute, Chongqing 401123, \\ China; qianwang@126.com \\ 3 Laboratory of Power Transmission Equipment \& System Security and New Technology, \\ Chongqing University, Chongqing 400044, China; cquhaojian@126.com \\ * Correspondence: tangchao_1981@163.com; Tel.: +86-023-68251265; Fax: +86-023-68251265
}

Academic Editor: Issouf Fofana

Received: 26 January 2017; Accepted: 17 March 2017; Published: 20 March 2017

\begin{abstract}
In this paper, polysiloxane is used to modify insulation paper cellulose, and molecular dynamics methods are used to evaluate the glass transition temperature and mechanical properties of the paper before and after the modification. Analysis of the static mechanical performance of the model shows that, with increasing temperature, the elastic modulus of both the modified and unmodified cellulose models decreases gradually. However, the elastic modulus of the modified model is greater than that of the unmodified model. Using the specific volume method and calculation of the mean square displacement of the models, the glass transition temperature of the modified cellulose model is found to be $48 \mathrm{~K}$ higher than that of the unmodified model. Finally, the changes in the mechanical properties and glass transition temperature of the model are analyzed by energy and free volume theory. The glass transition temperatures of the unmodified and modified cellulose models are approximately $400 \mathrm{~K}$ and $450 \mathrm{~K}$, respectively. These results are consistent with the conclusions obtained from the specific volume method and the calculation of the mean square displacement. It can be concluded that the modification of insulation paper cellulose with polysiloxane will effectively improve its thermal stability.
\end{abstract}

Keywords: insulation paper; polysiloxane; glass transition temperature; mean square displacement; molecular simulation

\section{Introduction}

The glass transition temperature of a polymer is very important, being the turning point at which a polymer material transitions from a glass state to a highly elastic state [1]. The main component of oil-immersed power transformer insulation paper is natural cellulose [2], which is composed of crystalline and amorphous regions. The crystalline region is closely arranged and its structure is stable, meaning that its performance is relatively stable under high temperatures. In contrast, the amorphous region is loosely and irregularly arranged, and so thermal aging usually starts from the amorphous region $[3,4]$. The initial mechanical properties of cellulose insulation paper can basically meet the needs of its applications, but after its glass transition temperature changes, its mechanical properties seriously decline [5], which causes the paper to fail to meet the needs of the application. The general operating temperature of a transformer is below $100^{\circ} \mathrm{C}$, but local winding temperatures can exceed $473 \mathrm{~K}$ under extreme conditions. At $473 \mathrm{~K}$, the properties of many polymers change greatly [6]; thus, the enhancement of the glass transition temperature of cellulose insulation paper is of important practical significance. 
Polysiloxane has many advantages, such as high temperature stability, oxidation resistance, and water repellency, and so has been used to modify the toughness and thermal stability of polymer in recent years [7-9]. Li et al. [10] used polysiloxane to graft and modify E-20 modified epoxy. Spectral and differential thermal analyses showed that the thermal stability of the modified resin was improved significantly. Su et al. [11] used dimethyl-diethoxy-silane to modify bisphenol A epoxy resin, and found that the tensile strength, elongation at break, and mechanical properties of the modified material were improved, and the glass transition temperature reached $166.07^{\circ} \mathrm{C}$. Zhang et al. [12] used polysiloxane to improve the thermal stability and mechanical properties of resin. Guan et al. [13] used fluorine-containing silicone to modify resin, and reported that the maximum thermo-gravimetric rate temperature of the modified composite material reached $375{ }^{\circ} \mathrm{C}$. Regarding other polymers, Zong et al. [14] used polysiloxane to modify polyurethane, and found that the water resistance properties of the modified material were improved. Obviously, modification with polysiloxane has a good effect on the performance of polymers, and especially has great potential for improving their thermal stability.

With the development of computer simulation technology, molecular simulation has been widely applied to the study of material properties [15-17]. Moreover, the results of studies combining molecular simulations and experiments have shown simulated and experimental values that were highly consistent, indicating that molecular simulation of materials is feasible and effective [18,19]. Molecular simulation has been widely used in researching the properties of insulation paper cellulose to enhance its thermal stability. Liao et al. [20] grafted dicyandiamide onto the C6 atoms of cellulose chains, and showed through molecular simulation that the stability of the modified cellulose chain in water and acid environment was improved. Cheng et al. [21] used inorganic particles to modify cellulose, and reported that the thermal stability of the modified cellulose was improved. However, the modification of insulation paper cellulose with polysiloxane has rarely been reported.

Therefore, this paper focuses on the two important indexes of the thermal stability of cellulose insulation paper: glass transition temperature and mechanical properties. The effect of polysiloxane on the thermal stability of insulation paper cellulose was evaluated by a molecular dynamics simulation method. The internal mechanism of the effect of polysiloxane on the mechanical properties, exercise intensity, and glass transition temperature of the cellulose was also studied.

\section{Model Building and Parameter Setting}

\subsection{Model Building}

Mazeau and Heux [22] used molecular simulation to study a model of the amorphous region of cellulose chains with different degrees of polymerization (DP), of 10, 20, and 40 . They found no obvious difference in molecular conformation or physical and chemical properties. Chen et al. [23] suggested that, for the amorphous models of cellulose with a $D P$ of 20 , the mechanic properties obtained by molecular simulation were consistent with the experimental data. Meanwhile, our previous research results $[24,25]$ also verified the consistency of molecular simulation and experiment results when $D P$ is 20. Therefore, in this paper, a cellulose chain with a $D P$ of 20 was chosen to build the model. The $D P$ of the polysiloxane used for grafting and modification was 3 . The polysiloxane is grafted onto the hydroxyl group of the $\mathrm{C} 6$ atom in the cellulose unit, which is shown in Figure 1. The model for the modified cellulose was established using the method used to construct the amorphous region model proposed by Theodorou and Wsuter [26]. The target density of the model was $1.5 \mathrm{~g} / \mathrm{cm}^{3}$. The constructed model is shown in Figure 2. 


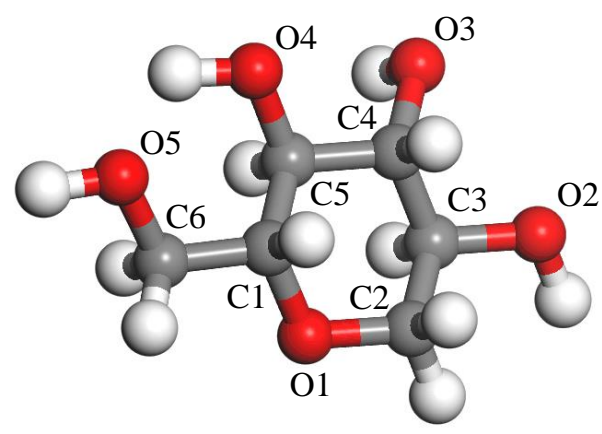

Figure 1. Unit of insulation paper cellulose.

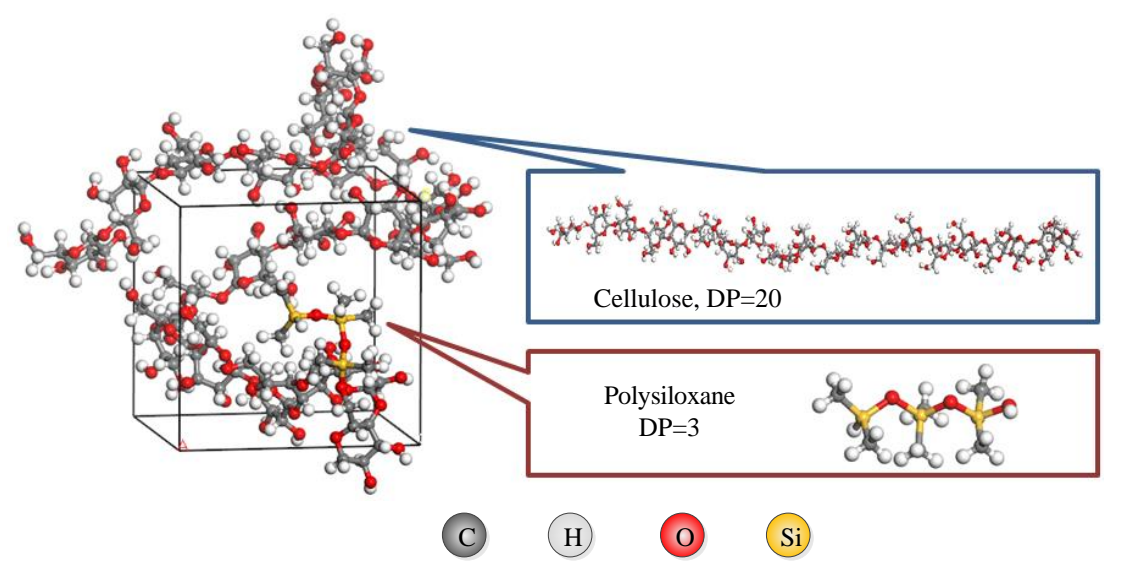

Figure 2. Amorphous model of cellulose modified by polysiloxane.

\subsection{Parameter Setting}

Structural optimization and energy optimization of the model was required. First, 5000 steps of energy minimization were carried out. The model was then structurally optimized through molecular dynamics simulation. The force field used in the structural optimization and molecular dynamics simulation was PCFF (polymer consistent forcefield) [27], which is very suitable for the treatment of carbohydrates and other organic molecules. During the structural optimization, dynamics simulations of 50 ps were carried out at temperatures of $1000 \mathrm{~K}, 800 \mathrm{~K}$, and $600 \mathrm{~K}$. Energy minimization was carried out after every dynamics simulation, and the obtained minimized structure was used as the initial conformation for the next dynamics simulation. Then, dynamics simulation of 50 ps and energy minimization were carried out at $400 \mathrm{~K}$. After the above processing, the local unreasonable structure in the amorphous model was basically eliminated, which made the model stable and closer to the real material, and provided a reasonable equilibrium geometry conformation for the next molecular dynamics simulation [28]. On this basis, the molecular dynamics simulation was the carried out. To determine the glass transition temperature before and after modification, a temperature range of $200-650 \mathrm{~K}$ was selected for the simulation, with every $50 \mathrm{~K}$ a target temperature. The molecular dynamics simulation of each target temperature was based on the fully optimized structure. First, equilibrium simulation of 100 ps was carried out, after which molecular dynamics simulation of $200 \mathrm{ps}$ was carried out. The integral step length was $1 \mathrm{fs}$, and the dynamic information of the system was collected every $5000 \mathrm{fs}$.

\section{Analysis of Simulation Results}

\subsection{Mechanical Properties}

The relationship between stress and strain can be obtained by the generalized Hooke's law: 


$$
\boldsymbol{\sigma}_{i}=\mathbf{C}_{i j} \varepsilon_{j}
$$

where $\mathbf{C}$ represents a $6 \times 6$ elastic coefficient matrix, $\varepsilon$ is the stress matrix, $\sigma$ is the strain matrix.

First, the inverse matrix $\mathbf{S}$ of the $\mathbf{C}$ matrix is obtained, and then the effective bulk modulus and shear modulus are calculated by Reuss' average method [29]:

$$
\begin{aligned}
& K=[3(a+2 b)]^{-1} \\
& G=\frac{5}{4 a-4 b+3 c}
\end{aligned}
$$

In the formula:

$$
\begin{aligned}
& a=\frac{1}{3}\left(\mathbf{S}_{11}+\mathbf{S}_{22}+\mathbf{S}_{33}\right), \\
& b=\frac{1}{3}\left(\mathbf{S}_{12}+\mathbf{S}_{23}+\mathbf{S}_{31}\right), \\
& c=\frac{1}{3}\left(\mathbf{S}_{44}+\mathbf{S}_{55}+\mathbf{S}_{66}\right) .
\end{aligned}
$$

Through the relationship between the modulus of the same-phase material:

$$
E=2 G(1+v)=3 K(1-2 v),
$$

where $E$ represents the elastic modulus, $K$ is the volume modulus, $G$ is the shear modulus, and $v$ represents Poisson's ratio.

The ratio of strain and stress is elastic modulus $E$, which reflects the rigidity of a material. The greater the value of $E$ is, the greater the rigidity and the stronger the ability of the material to resist deformation will be. Thus, $E$ can reflect the macro-mechanical strength of transformer insulation paper [30,31]. The elastic modulus calculated for the modified model and unmodified model at different temperatures is shown in Figure 3.

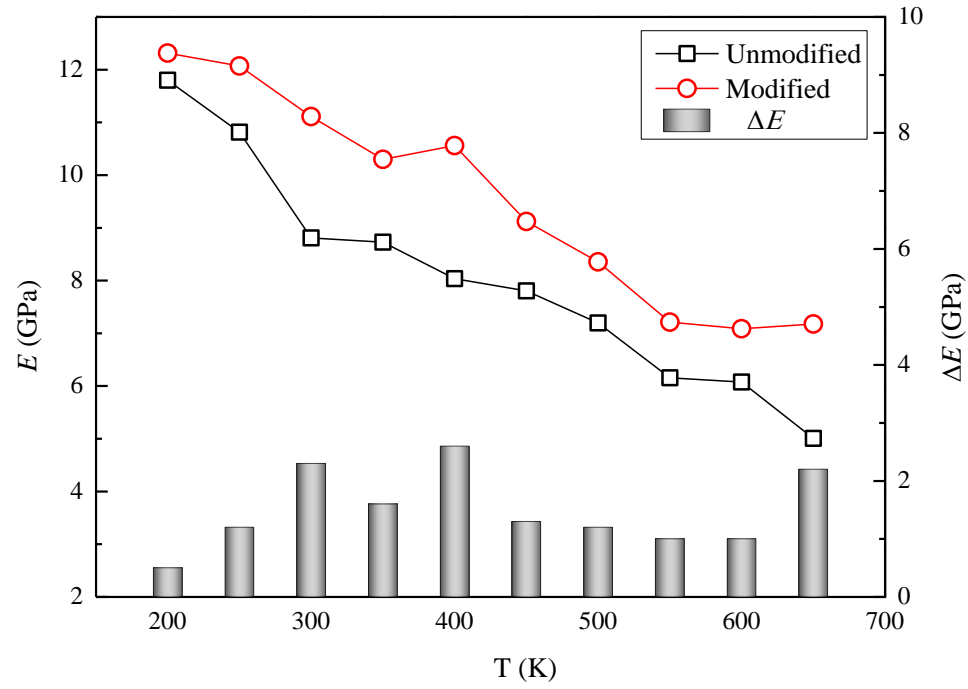

Figure 3. Elastic modulus of modified and unmodified models.

With increasing temperature, the elastic modulus of both models gradually decreases. However, the elastic modulus of modified model is larger than that of the unmodified model in the whole simulation process. In Figure 3, $\Delta E$ is the difference between the elastic modulus of the modified model and the elastic modulus of the unmodified model. $\Delta E$ is positive in the whole temperature range, which shows that the elastic modulus of the modified model is improved over that of the 
unmodified model. This is because, when modifying cellulose by polysiloxane grafting, the bond energy of the flexible $\mathrm{Si}-\mathrm{O}$ bond introduced on the cellulose chain is $451 \mathrm{~kJ} / \mathrm{mol}$, which is greater than the $356 \mathrm{~kJ} / \mathrm{mol}$ of the $\mathrm{C}-\mathrm{C}$ bond. The greater the bond energy, the harder it is to break the bond, so the elastic modulus of the model modified with polysiloxane is always higher than that of the unmodified model with increasing temperature. This means that the deformation resistance, mechanical properties, and thermal stability of the model modified with polysiloxane are enhanced.

\subsection{Glass Transition Temperature}

As its temperature is gradually increased, an amorphous material will undergo glass transition. The transition temperature [1] of a material from a glass state to a highly elastic state is known as the glass transition temperature $T_{\mathrm{g}}$. If the temperature continues to rise, a polymer material will change from a highly elastic state to a viscous flow state at its melting point, $T_{\mathrm{f}}$. Generally, the properties of polymers are stable before glass transition. At temperatures higher than the glass transition temperature, the mechanical properties of polymers, namely mechanical strength and thermal stability, will be significantly reduced. Therefore, the improvement of the glass conversion properties of insulation paper cellulose at high temperature is significantly important.

Among the methods used to evaluate the glass transition temperature of polymers, the most common and reliable is the volume-temperature curve method [32,33]. In the NPT ensemble (fixed values of particle number $N$, pressure $P$, and temperature $T$ ), molecular simulation was performed on the models at each selected temperature to calculate the density parameter. The reciprocal of density is the specific volume, thus, the figure of specific volume versus temperature can be obtained. Next, the intersection of lines fitting the regions before and after the inflection point of the specific volume temperature is the glass transition turning point. Thus, the corresponding temperature is the glass transition temperature. The glass transition temperature fitting curve is shown in Figure 4.

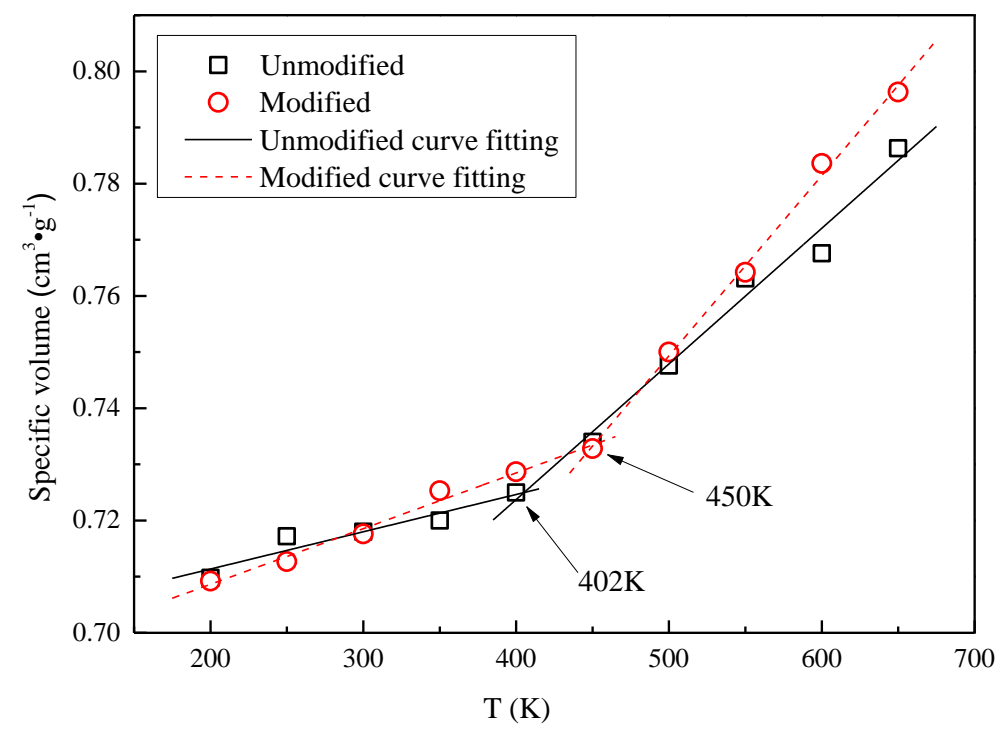

Figure 4. Specific volume-temperature curve.

The glass transition temperature of the unmodified model is $402 \mathrm{~K}$, while that of the model modified with polysiloxane is $450 \mathrm{~K}$, which means that the modification improves the glass transition temperature of the cellulose chain by $48 \mathrm{~K}$. In the Handbook of Polymers [34], the glass transition temperature of variety of industrial cellulose is reported to be between 250 and $580 \mathrm{~K}$, but this is only a reference. Thus, the glass transition temperatures of the simulated cellulose and modified cellulose models in this paper are credible, and the comparative analysis of the glass transition temperature of the modified and unmodified model is valid. 
The glass transition temperature of the modified model is higher than that of the unmodified model, which means that the process from the glassy state to highly elastic state of the modified model is slower than that of the unmodified model. Therefore, the modification of cellulose should result in excellent performance and enhanced thermal stability.

\subsection{Glass Transition Temperature Analysis}

The more intense the chain movement in insulation paper cellulose, the worse its mechanical performance, which leads to a poor thermal stability. The intensity of the chain movement can be analyzed by the mean square displacement $(M S D)$. The MSD describes the overall movement of the molecular chain centroid [35], and can be calculated as:

$$
M S D=\left\langle\left|\vec{r}_{i}(t)-\vec{r}_{i}(0)\right|^{2}\right\rangle,
$$

where $\vec{r}_{i}(t)$ and $\vec{r}_{i}(0)$ represent molecular or atomic position vectors at time $\mathrm{t}$ and initial time $\mathrm{i}$, respectively, and $<>$ represents the ensemble average. The MSD of the modified model and the unmodified model are shown in Figure 5.
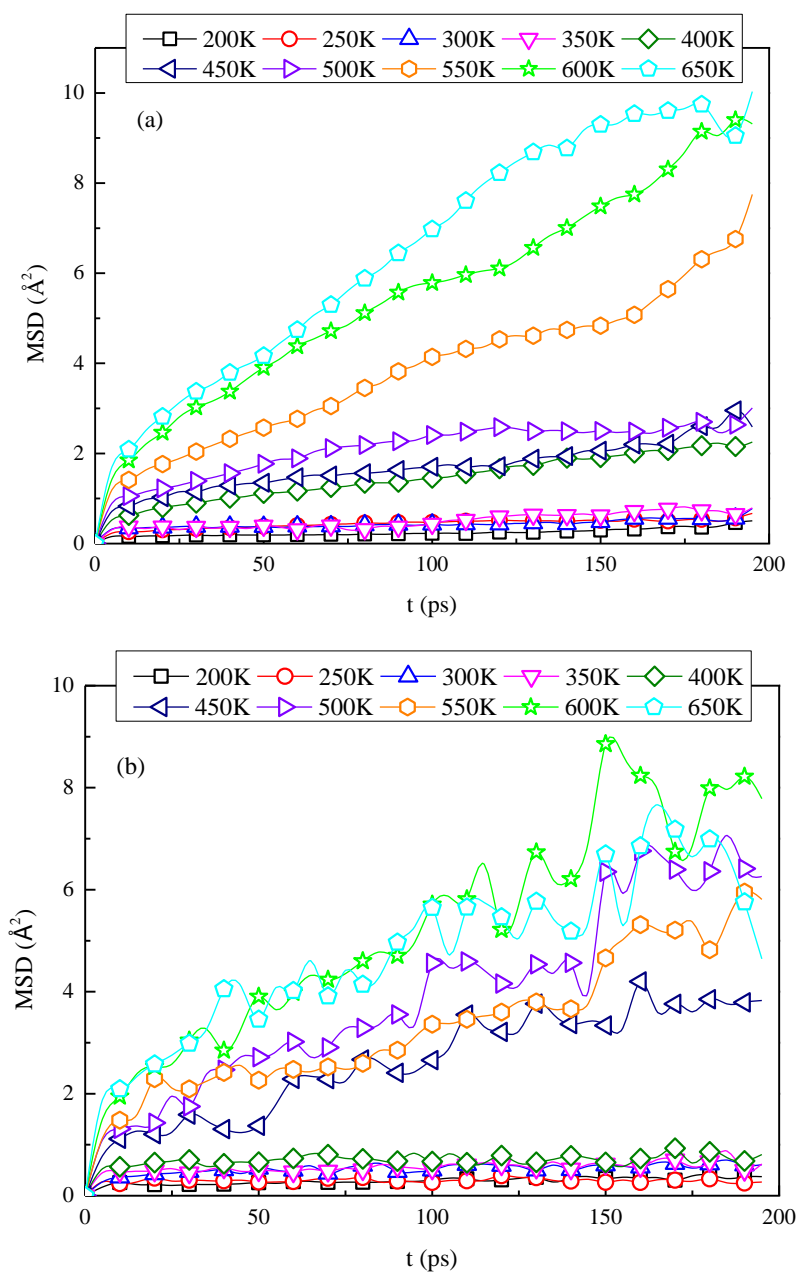

Figure 5. MSD of (a) unmodified model and (b) modified model at different temperatures.

According to Figure 5, the degree of chain movement increases gradually with temperature, which indicates that insulation paper cellulose is affected by temperature during transformer operation. In the unmodified model, between 350 and $400 \mathrm{~K}$, the MSD of the chain appears to jump, which is basically 
consistent with the glass transition temperature of the unmodified model of $402 \mathrm{~K}$. Between 500 and $550 \mathrm{~K}$, the $M S D$ of the unmodified model appears to jump again, which may correspond to the transition temperature of cellulose chain from the highly elastic state to the viscous flow state. In the modified model, the first jump in the MSD of the chain between 400 and $450 \mathrm{~K}$ also corresponds to the glass transition temperature $(450 \mathrm{~K})$ of the modified model. The MSD of the modified model does not appear to jump again obviously within the studied temperature range. This may be because the modification of the cellulose chain not only increased the glass transition temperature of the chain, but also increased the temperature of the highly elastic state to the viscous flow state transition. It can also be seen that, before the glass transition, the intensity of the movement of the chain was relatively small, and the MSD value of the two models is less than $1 \AA^{2}$. After the glass transition, the intensity of the movement of the chain is obviously enhanced; the maximum MSD of the unmodified model is about $10 \AA^{2}$, while that of the modified model is about $9 \AA^{2}$.

The chain movement is the direct embodiment of the thermal movement ability of the insulation paper cellulose chain. The more intense the chain movement is, the worse the mechanical performance will be, which leads to a poor thermal stability. The thermal stability of insulation paper cellulose is improved by grafting with polysiloxane. In oil-immersed transformers, cellulose insulation paper is wound around the copper conductor as electrical insulation. If the mechanical properties of the insulation paper are affected by temperature and become worse, the insulation paper wrapped outside the copper wire will be easily damaged when the conductor coil is subject to mechanical stress, which will damage the insulation of the transformer. Therefore, the improvement of the mechanical properties of insulation paper also indirectly improves the insulation of the transformer, thus, ensuring its safe operation.

\section{Analysis and Discussion}

\subsection{Enhancement Mechanism}

Polysiloxane is a cross-linked polymer with $\mathrm{Si}-\mathrm{O}-\mathrm{Si}$ as the main chain, and silicon atoms connected to other organic groups. The organic groups can be methyl or phenyl, so polysiloxane can have organic and inorganic characteristics. Polysiloxane has many excellent properties, such as heat resistance and chemical corrosion resistance. The $\mathrm{Si}-\mathrm{O}-\mathrm{Si}$ bond is the basic bond form of polysiloxane, essentially the same as quartz, but its side groups are connected to organic groups. The thermal stability of inorganic compounds composed of $\mathrm{Si}-\mathrm{O}$ bonds can be as high as $2073 \mathrm{~K}$. When organic substituents are introduced into the polymer, its thermal stability will decrease by $623-873 \mathrm{~K}$, but its heat resistance will still be better than that of common organic compounds. Additionally, because of its organic and inorganic characteristics, it can solve compatibility problems well. The bond energy of the $\mathrm{Si}-\mathrm{O}$ bond is $451 \mathrm{~kJ} / \mathrm{mol}$, much higher than the energy of the $\mathrm{C}-\mathrm{C}$ bond of about $356 \mathrm{~kJ} / \mathrm{mol}$. Thus, the thermal stability of the $\mathrm{Si}-\mathrm{O}$ bond is better than that of the $\mathrm{C}-\mathrm{C}$ bond. The length of the $\mathrm{Si}-\mathrm{O}-\mathrm{Si}$ bond is also longer $(0.164 \mathrm{~nm})$, and its bond angle is larger $\left(140-180^{\circ}\right)$ [36]. The chain structure of polysiloxane is very soft, the interaction between chains is weak, and its surface tension is low, which means that polysiloxane can still maintain its original performance in a wide range of temperatures. Therefore, the mechanical properties of cellulose modified with a certain amount of polysiloxane change little with increasing temperature, resulting in a very good thermal stability.

Energy is a parameter that can best reflect the stability of the system. The energy of the system under a PCFF force field [6] can be expressed as:

$$
E_{\text {total }}=\left(E_{\text {internal }}+E_{\text {cross }}\right)+E_{\text {nobond }}
$$

where $E_{\text {internal }}+E_{\text {cross }}$ represents the bond energy and $E_{\text {nobond }}$ represents non-bond energy. Figure 6 shows the overall potential energy and non-bond energy of the unmodified model and modified model at each temperature studied in this paper. 

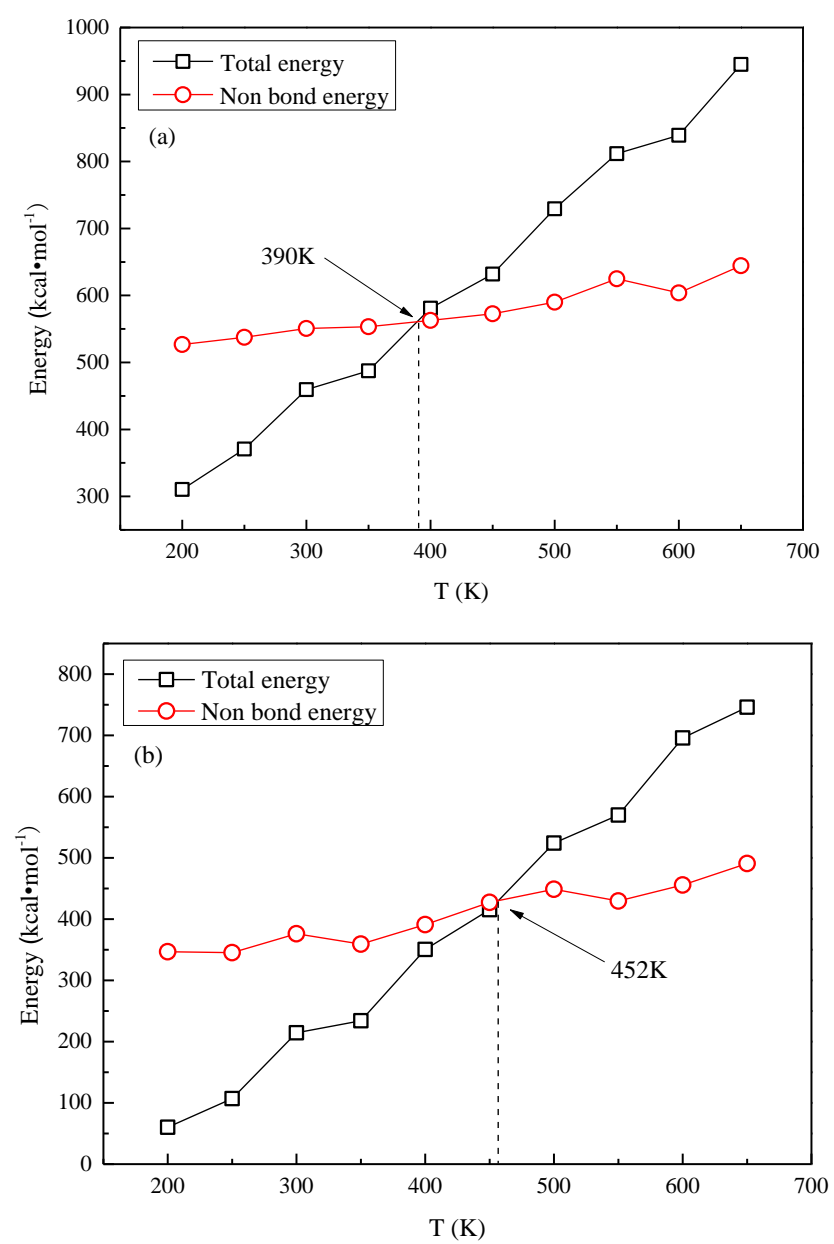

Figure 6. Energies of the two models: (a) the unmodified model; and (b) the modified model.

The overall potential energy and the non-bond energy of both models increase linearly, but the growth rate of the non-bond energy is obviously lower than that of the overall potential energy. In the unmodified model, the overall potential energy starts to become larger than the non-bond energy at $390 \mathrm{~K}$. In the modified model, the overall potential energy starts to become larger than the non-bond energy at about $452 \mathrm{~K}$, a difference of about $62 \mathrm{~K}$. Equation (6) shows that when the value of the overall potential energy starts increase beyond that of the non-bond energy, the value changes from negative to positive. This indicates that the repulsive force between atoms is greater than the attraction force between atoms in the interior of the molecule, and the chemical bond becomes unstable. As the temperature is gradually increases, some active bonds and glycosidic bonds become easy to break and, thus, thermal degradation of the cellulose occurs. Upon thermal degradation, the $D P$ of the cellulose will decrease, resulting in decreased mechanical properties of the cellulose insulation paper.

\subsection{Mechanism of Effect of Polysiloxane on Chain Movement Intensity and Glass Transition Temperature of Cellulose}

The free volume of a material has a great influence on its glass transition temperature. According to the free volume theory proposed by Fox and Flory [37], the volume of a polymer is composed of the free volume occupied and unoccupied by the polymer. Due to the difference in the expansion coefficient, when the temperature increases, the volume of the polymer increases linearly, and the free volume will jump near the glass transition temperature [30]. In this paper, the mechanism of the change in the glass transition temperature is analyzed using the free volume theory from a microscale perspective. The hard sphere probe method was used to calculate the size distribution of the free volume in 
the model, and the probe radius was set to $1 \AA$ A. Figure 7 shows the free volume distribution of the unmodified model and modified model, in which the blue part is the distribution of the free volume.
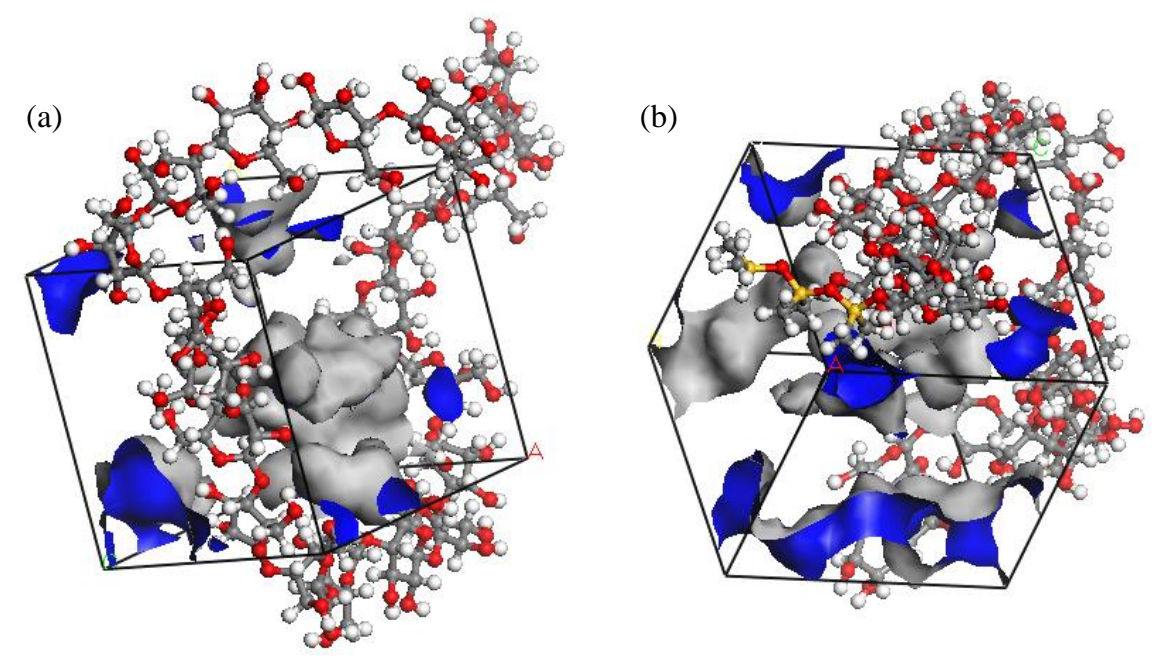

Figure 7. Free volume of the two models: (a) the unmodified model; and (b) the modified model.

Figure 8 shows the free volume fitting curve of the unmodified model and modified model. In the unmodified model, the free volume changes little below a temperature of $392 \mathrm{~K}$. At $392 \mathrm{~K}$, the free volume jumps and then gradually increases. In the modified model, the free volume changes little below $440 \mathrm{~K}$, and then gradually increases over $440 \mathrm{~K}$. An increase in free volume increases the space for the movement of the cellulose chain. When the molecular chain starts to move, the cellulose changes from the glass state to the highly elastic state. After conversion to the high elastic state, the mechanical properties of the cellulose chain decrease dramatically, and its thermal stability also decreases. From the present free volume theory analysis, the difference of $48 \mathrm{~K}$ between the jump in the free volume of the unmodified model and modified model is basically consistent with the conclusions obtained from the previous specific volume method analysis. Therefore, it is confirmed that polysiloxane modification will enhance the thermal stability of cellulose.

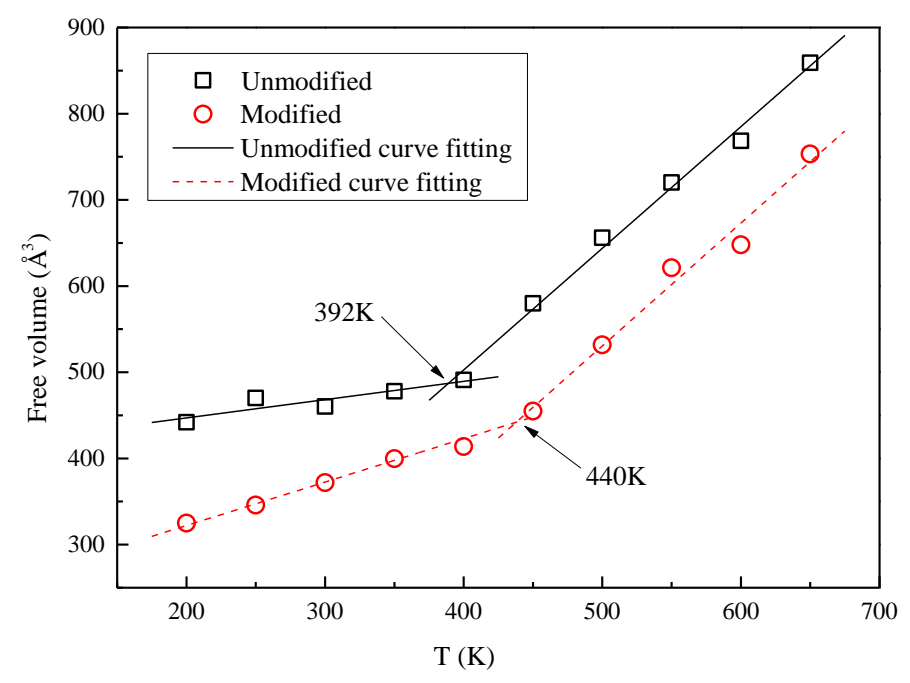

Figure 8. Fitting curve of free volume. 


\section{Conclusions}

In this paper, the molecular dynamics method is applied to evaluate the glass transition temperature, mechanical properties, and chain movement intensity of insulation paper cellulose before and after modification with polysiloxane. Through comparison of the model data, the following conclusions are obtained:

(1) For the modified cellulose insulation paper, the anti-deformation energy of the cellulose is increased, and its mechanical properties are improved.

(2) Based on the specific volume method, the glass transition temperature of the modified cellulose model increased by $48 \mathrm{~K}$ than that of the unmodified model. When the temperature increases near the glass transition temperature, the mean square displacement of the two models appears to jump, and the chain movement of the insulation paper cellulose becomes intense.

(3) The mechanism of the change in the mechanical properties of the model is discussed based on energy. The glass transition temperature range obtained from the free volume theory is basically the same as that obtained by the specific volume method. Therefore, the modification of insulation paper cellulose by polysiloxane grafting will greatly enhance the thermal stability of insulation paper.

Acknowledgments: The authors wish to thank the scientific project funded by the State Grid Chongqing Electric Power Cooperation Chongqing Electric Power Research Institute, and the Fundamental Research Funds for the Central Universities (Grant No. XDJK2014B031) for their financial support.

Author Contributions: Chao Tang, Song Zhang conceived and designed the simulations; Song Zhang and Xiaobo Wang performed the simulations; all authors analyzed the data; Chao Tang and Song Zhang wrote the paper, while other authors offered their modification suggestions for the manuscript.

Conflicts of Interest: The authors declare no conflict of interest.

\section{References}

1. Ke, Y.C.; He, P.S. Macromolecule Physical Tutorial; Chemistry Industry Press: Beijing, China, 2006; pp. 157-161.

2. Liu, R.Q. Basis of Cellulose Chemistry; Science Press: Beijing, China, 1985; pp. 115-123.

3. Zhu, M.Z. Molecular Dynamics Study of Thermal Aging of Oil-impregnated Insulation Paper. Ph.D. Thesis, Chongqing Univeristy, Chongqing, China, April 2012.

4. Yan, J.Y.; Wang, X.L.; Li, Q.M.; Zhou, Y.; Wang, Z.D.; Li, C.R. Molecular dynamics simulation on the pyrolysis of insulation paper. Proc. CSEE 2015, 35, 5941-5949.

5. Yang, T. Molecular Dynamics Study on the Impact of Temperature and Electric Field on the Microscopic Properties of Oil-Immersed. Ph.D. Thesis, Chongqing Univeristy, Chongqing, China, May 2013.

6. Wang, Y.Y.; Yang, T.; Liao, R.J.; Zhang, D.W.; Liu, Q.; Tian, M. Glass Transition in amorphous region of transformer insulation paper by molecular dynamics. High Volt. Eng. 2012, 31, 1199-1206.

7. Li, X.M.; Pan, Q.; Lin, Y.D.; You, B.; Sun, Y.J. Synthesis and Characterization of epoxy/poly siloxane hybrid materials. J. Chongqing Univ. 2011, 34, 112-122.

8. Zhao, S. Study on Preparation and Properties of Polysiloxane Modified Epoxy Encapsulating Material. Ph.D. Thesis, Harbin University of Science and Technology, Harbin, China, March 2014.

9. Zhang, H.Z. Study on Polysiloxane Copolymerization Modified Epoxy Resin Capability. Ph.D. Thesis, Chongqing University, Chongqing, China, May 2008.

10. Li, Y.W.; Shen, M.M.; Huang, H.Y.; Ma, Y.J.; Ha, C.Y. Synthesis and properties of polyphenylsilicone and polymethylphenylsilicone modified epoxy resins. Acta Polym. Sin. 2009, 11, 1086-1090. [CrossRef]

11. Su, Q.Q.; Liu, W.Q.; Wang, W.R.; Liu, Y.F. Investigation on epoxy resin modified by diethoxydimethylsilane. Chem. Mater. Constr. 2008, 24, 23-25.

12. Zhang, S.; Xie, J.L.; Deng, L.J. Study on organosilicon modified epoxy resin adhesive for heat-resistance. Mater. Rev. 2006, 20, 23-25.

13. Guan, D.B.; Cai, Z.Y.; Fang, C.; Qiu, X.M.; Dou, Y.L. Preparation and properties of fluorinated silicone modified epoxy resin composite. Polym. Mater. Sci. Eng. 2015, 31, 120-125. 
14. Zong, J.P.; Zhang, Q.S.; Li, J.S.; Sun, H.F.; Yu, Y.T.; Liu, S.J.; Liu, Y.H. Comparative of polydimethylsiloxane modified graft and block polyurethane dispersions. Polym. Mater. Sci. Eng. 2011, 27, 62-65.

15. Sun, W.F.; Wang, X. Molecular dynamics simulation study of polyimide/copper-nanoparticle composites. Acta Phys. Sin. 2013, 62, 186202.

16. Lin, C.P.; Liu, X.J.; Rao, Z.H. Molecular dynamics simulation of the thermophysical properties and phase change behaviors of aluminum nanoparticles. Acta Phys. Sin. 2015, 64, 083601.

17. Kopper, K.P.; Küpper, D.; Reeve, R.; Mitrelias, T.; Gunn, D.S.D.; Jenkins, S.J. Chemically selective modification of spin polarization in ultrathin ferromagnetic films: Microscopic theory and macroscopic experiment. Phys. Rev. B 2009, 80, 1956-1960. [CrossRef]

18. Yu, S.; Yang, S.; Cho, M. Molecular dynamics study to identify mold geometry effect on the pattern transfer in thermal nanoimprint lithography. Jpn. J. Appl. Phys. 2009, 48. [CrossRef]

19. Zhu, M.Z.; Chen, Y.F.; Gu, C.; Liao, R.J.; Zhu, W.B.; Du, X.M. Simulation on thermodynamic properties of amorphous cellulose based on molecular dynamics. High Volt. Eng. 2015, 41, 432-439.

20. Liao, R.J.; Nie, S.J.; Zhou, X.; Wang, K.; Yuan, L.; Yang, L.J.; Cheng, H.C. Molecular dynamics simulation on the hydrophilicity of physical modification cellulose insulating materials. Proc. CSEE. 2013, 39, 1-7.

21. Cheng, W.; Wang, K.; Fu, Q. Preparation of inorganic particle grafted cellulose fibre and its thermostability. Plast. Ind. 2014, 42, 113-117.

22. Mazeau, K.; Heux, L. Molecular dynamics simulations of bulk native crystalline and amorphous structures of cellulose. J. Phys. Chem. B 2003, 107, 2394-2403. [CrossRef]

23. Chen, W.; Lickfield, C.G.; Yang, Q.C. Molecular modeling of cellulose in amorphous state. Part 1: Model building and plastic deformation study. Polymer 2004, 45, 1063-1071. [CrossRef]

24. Tang, C.; Zhang, S.; Zhang, F.Z.; Li, X.; Zhou, Q. Simulation and experimental about the thermal aging performance improvement of cellulose insulation paper. Trans. China Electrotech. Soc. 2016, 31, 68-76.

25. Tang, C.; Zhang, S.; Li, X.; Xiong, B.F.; Xie, J.T. Experimental analyses and molecular simulation of the thermal aging of transformer insulation paper. IEEE Trans. Dielectr. Electr. Insul. 2015, 22, 1131-1138. [CrossRef]

26. Theodorou, D.N.; Wsuter, U. Detailed molecular structure of a vinyl polymer glass. Macromolecules 1985, 18, 1467-1478. [CrossRef]

27. Sun, H. Ab-initio calculations and force field development for computer simulation of polysilanes. Macromolecules 1995, 28, 701-712. [CrossRef]

28. Tao, C.G.; Feng, H.J.; Zhou, J.; Lv, L.H.; Lu, X.H. Molecular simulation of oxygen adsorption and diffusion in polypropylene. Acta Phys. Chim. Sin. 2009, 25, 1373-1378.

29. Watt, J.P.; Davies, G.F.; O'Connell, R.J. The elastic properties of composite materials. Rev. Geophys. Space Phys. 1976, 14, 541-563. [CrossRef]

30. Tanaka, F.; Iwata, T. Estimation of the elastic modulus of cellulose crystal by molecular mechanics simulation. Cellulose 2006, 13, 509-517. [CrossRef]

31. Zhang, S.; Tang, C.; Chen, G.; Zhou, Q.; Lv, C.; Li, X. The influence and mechanism of nano $\mathrm{Al}_{2} \mathrm{O}_{3}$ to the thermal stability of cellulose insulation paper. Sci. Sin. Techol. 2015, 45, 1167-1179.

32. He, P.S. The Mechanical Properties of High Polymer; Press of University of Science and Technology of China: Hefei, China, 2008; pp. 185-196.

33. Fu, Y.Z.; Liu, Y.Q.; Lan, Y.H. Molecular simulation on the glass transition of polypropylene. Polym. Mater. Sci. Eng. 2009, 25, 53-56.

34. Brandrup, J.; Immergut, E.H.; Grulke, E.A. Polymer Handbook; Wiley-Interscience Publication: New York, NY, USA, 1999; pp. 476-479.

35. Mazur, P.; Maradudin, A.A. Mean-square displacements of atoms in thin crystal films. Phys. Rev. B 1981, 24, 2996-3007. [CrossRef]

36. Hao, Z.F.; Zhang, J.; Wu, Y.H.; Yu, J.; Yu, L. Synthesis and thermal stability properties of boron-doped silicone resin. J. Appl. Polym. Sci. 2014, 131, 1366-1373. [CrossRef]

37. Fox, T.G.; Flory, P.J. Second-order transition temperatures and related properties of polystyrene. I. influence of molecular weight. J. Appl. Phys. 1950, 21, 581-591. [CrossRef]

(C) 2017 by the authors. Licensee MDPI, Basel, Switzerland. This article is an open access article distributed under the terms and conditions of the Creative Commons Attribution (CC BY) license (http:/ / creativecommons.org/licenses/by/4.0/). 